\title{
A Leap Forward Path Model of Niche based on Brand Ecological Theory
}

\author{
Christine Goddard ${ }^{1}$ and Cristopher Aschemann-Witzel ${ }^{2}$ \\ ${ }^{1,2}$ Concordia University, Canada \\ ${ }^{1}$ christine.goddard@encs.concordia.ca
}

\begin{abstract}
Based on the theory of brand ecology and evolutionary economics, this paper constructs a model of the transition path of brand niche and clarifies the driving mechanism of the transition of brand niche from the perspective of consumer cognition, that is, brand entrepreneurs under the leading role of heterogeneous human capital Bring out the innovative spirit of brand entrepreneurs through the three elements of property rights, system, and culture, and follow satisfactory decisions to search for consumer cognition to form value co-creation (value niche). Relying on the mobile phone industry, a consumer cognitive niche system has been constructed and the importance of various factors affecting the industry niche has been identified. Specifically, the mobile phone brand niche system is composed of 12 factors at the product level, brand level, and industry level. Among them, the key factors mainly include "quality perception, performance perception, functional innovation, brand reputation, and technological change." The high factor weight is a key factor that affects consumer brand perception and a key indicator that determines the transition of the ecological niche. Taking the Apple mobile phone as a specific case, the niche at each time point was measured and the niche transition was proved to be a historical process, and the comprehensive evaluation scores of the niche of 6 mobile phones were calculated and ranked. The results of the study show that the overall trend is rising first and then stabilizing.
\end{abstract}

Keywords: Consumers, Brand ecology theory, Niche advancement, Brand awareness

\section{Introduction}

At present, the definition of the concept of transition in academia has not yet been unified. In physics, a transition is a process in which the state of a quantum mechanical system undergoes a jump change. The transition follows the evolution process of the law of hereditymutation-natural selection. From the result of material form, qualitative change occurs. The basis of evolution is "gene". There are several representative classifications of transition methods: the first type is energy level transition [1] and organizational transition [2]. Energy level transition is to observe the change of the state from a static perspective (such as the state from 0 to 1 , from nothing to something). Organizational transition emphasizes organizational learning and organizational change, which is essentially the embodiment of initiative. The second category is strategic transition and tactical transition. The strategic transition integrates the goals, missions, and visions within the organization, and is a long-term transition. The tactical transition reflects the company's short-term goals. The third category is divided into vertical transition and horizontal transition according to the direction of the transition.

Article history:

Received (October 9, 2020), Review Result (November 26, 2020), Accepted (January 6, 2021) 
Longitudinal transitions are up and down transitions, and horizontal transitions are parallel transitions [3]. The fourth category is active transition and passive transition from the perspective of exogenous and endogenous variables. Active transition emphasizes subjective initiative. People's thoughts and spirit are materialized in products and brands, and the form of expression is the optimization of old brands and old products. Passive transition emphasizes the impact of exogenous environmental impact on the initiative, which is manifested in making good old brands and old products.

Regarding the brand niche, the marketing community will discuss the principles [4], the dimensions of specific brand composition and evaluation [5], crisis management, and countermeasures [6]. From the content point of view, it is what (what), but as to why (Why and how are not explained in the literature. The niche transition is summarized from the perspective of value chain reconstruction, and the sub-subjects are summarized from the perspective of enterprises [7] and high-tech industries [3]. However, the literature fails to further explain the path and law of the transition from the perspective of historical evolution and the macro level of institutional change.

Nelson and Winter created the paradigm of evolutionary economics in the 1960s, integrating the production of knowledge, diversification of things, variation, and market selection into the analytical framework of evolutionary theory. The evolution in marketing is niche overlap, movement, scaling, specialization, and generalization. In essence, evolution has the characteristic of continuity, the transition has the characteristic of discontinuity, evolution is the process segment of transition, and the transition is the inevitable result of evolution. This article combines the rational hypothesis of brand niche theory and evolutionary economics theory and tries to construct a brand niche transition model from the perspective of consumer cognition, to explain the "motivation" of the transition.

\section{Theoretical basis}

This research is mainly based on brand niche theory and evolutionary economics theory. An important theoretical basis of this article is the brand niche theory. From the perspective of the meaning of brand niche, the niche is the overall state of the brand using various resources in the system at a certain position in the niche system [8][9], and its connotation is the integration of all the living conditions of the brand [10]. From the perspective of the components of brand niche, Jarvis et al. focused on the perspective of customer preference and identified the two major components of customer loyalty and product involvement [11]. Foydel has built an ecological niche system composed of three elements: customers, channels, and suppliers [12]. From previous studies, the research on the brand niche is still in the stage of static exploration, but few scholars have been involved in the research on the transition of niche, especially the transition of brand niche. To make up for the deficiencies of the existing ecological niche research, this paper introduces the rational hypothesis of evolutionary economics theory to explore the internal driving mechanism of the brand niche transition and provide a theoretical basis for the industry. The main idea is to promote "the creation of novelty" [13] that is, innovation is the first principle of evolution. The driving force of evolution is two mechanisms: one is the learning and innovation mechanism. Learning is the process of knowledge absorption. Innovation comes from discoveries and is the reproduction of knowledge. This mechanism produces maternal diversity and is the main cause of evolution. The second is the environmental selection mechanism. This mechanism determines the form of diversity, considering the essential issues of "going" and "staying". The basis of evolution is the principle of "conventional error-search-environment", and the path includes path dependence and path 
creation. Another important theory in evolutionary economics is the constructivist theory of institutional change. The brand niche theory is used in the brand niche under different systems or the transition of the brand niche is the result of the free allocation of resources by the market. Constructivism is divided into liberalism and authoritarianism. Liberalism advocates the construction of systems consciously and rationally, and authoritarianism advocates the construction of systems by authoritative institutions (states). This article believes that liberal constructivism is more in line with the transition of brand niche, that is, it tends to induce the transition of brand niche through institutional design.

\section{Based on the model of the transition of consumer brand cognitive niche in the framework of evolutionary economics}

\subsection{The theory of evolutionary economics is embedded in the theory of brand niche: Theoretical basis}

Brand niche theory satisfies the three conditions of evolutionary economics theory: one is the life characteristics of the brand; the other is the humanized characteristics of the brand; the third is the survival characteristics of the brand.

(1) The vital characteristics of the brand. The niche characteristics of a brand determine the characteristics of life, and it is a collection of resources and environmental variable selection ranges [14] (resources are mainly affected by suppliers, distributors, and customers. The environment is the overall social environment). From a theoretical point of view, the niche theory conforms to the two basic elements of Darwin's theory of biological evolution, namely resources and environment. Evolution is the natural reproduction of organisms, and the gene of evolution is the interaction and fusion of resources and the environment. Evolutionary economics studies the evolution of organizations and regards the organization as a complex with life characteristics and basic functions derived from nature (self-organization phenomenon). The two theories are consistent in their ideological sources.

(2) The human characteristics of the brand. Brand personality is defined as a series of personality characteristics connected with the brand [15]. Brand personality is an internal factor that reflects the brand image, and the brand image is an important part of brand equity and an important source of consumer perceived value. Based on the analysis of brand image and consumer group characteristics, brand entrepreneurs can shape different brand personalities to match the ideal brand image in the minds of consumers. Evolutionary economics promotes learning and innovation. It is a conscious agency of people and a positive reinforcement mechanism for the development of organizations. Both theories emphasize human behavior at the level of motivation.

(3) The market characteristics of the brand. The resource attribute and competitive attribute of a brand determine the characteristics of the market. The connotation of both theories is a market selection mechanism for the survival of the fittest. The underlying factors that determine this mechanism are the flow of various energy. Brand development is played an important role by "flow", and it is metabolized through various "flows". Resource attributes are reflected by many "resource flows" such as information flow, technology flow, and capital flow. When many brands consume a certain kind of "flow", competition appears [16]. 


\subsection{Element components and models}

This article constructs a brand niche transition model from the perspective of consumer cognition based on the above-mentioned related theoretical foundations.

(1) Significance of historical evolution: transitions are caused by changes in factors that accompany the passage of time. The brand niche transition cannot be accomplished overnight but is a historical process. The path creation, dependence, lock-in, and the transition of brand niche produced in the long river of history are brand entrepreneurs who refer to the search criteria, that is, to make "correction" and "trial and error" after "correcting" and "trial and error" the process of.

(2) Search criteria: satisfactory decision. The management master Drucker draws on Simon's bounded rationality to make satisfactory decisions, which is different from "complete rationality". From the perspective of information economics, there is a phenomenon of information asymmetry. Brand entrepreneurs cannot fully obtain all the information that consumers recognize. Search cannot be unlimited. The principle of satisfactory decisionmaking must be followed. The frequency of search is restricted by satisfactory decisionmaking.

(3) Search the subject and the basis of development. The main body of the search is the brand entrepreneur (brand manager, designer, technical researcher, etc.), which is a community of interests. Brand building is the division of labor and collaboration between the different entities of the brand entrepreneur, and the interests of the business are the same and the risks are shared. The foundation of development has three elements: system, property rights, and culture, which are the three cornerstones for brand entrepreneurs to rise from "knowledge" (ideological convention) to "action" (behavior convention). From the perspective of consumer cognition, consumer cognition changes in different environments, and consumer cognition can change the existing knowledge structure of brand entrepreneurs and is the source of inspiration. The reason is that brand managers obtain part of the first-hand information through satisfactory decisionmaking and pass it on to designers. Designers continue to learn and innovate. Technologists industrialize and commercialize discoveries, and finally feedback to consumers to form value co-creation. From the element level of the unity of knowledge and action, property rights have two meanings here: one is brand ownership, management rights, income rights, and intellectual property rights based on the brand in terms of structure; the other is incentives for increasing returns based on property rights. The impact of culture on the integration of knowledge and action for brand entrepreneurs can be divided into three levels: macro, medium, and micro. The first is macro culture, which is shaped by factors such as history, national values, and systems. The second is regional culture, which is a specific manifestation of macro culture in a certain area, which is related to geographical location and regional traditions. The regional culture forms the brand regional culture, which is the local relationship after the brand is socialized, including the relationship between the human network, the earth, and the sky [22]. The third is corporate culture, which derives from corporate management culture and corporate brand culture. The Corporate management culture is a reflection of managers' values and outlook on life. Corporate brand culture is the collaboration between brand entrepreneurs' pursuit of spiritual value orientation with consumers and a process in which brand entrepreneurs and consumers complete brand resonance. From the perspective of brand entrepreneurs, brand

culture has an impact on brand entrepreneurs and makes them realize that maintaining consumers and maintaining high customer loyalty is the core of marketing. The system is 
constructed through a contract, ranging from the market allocation of resources system and property rights system to the small enterprise management system and brand building system.

(4) Brand ecosystem: the external environment and internal niche [17]. The external environment is economic, natural, political, technological, industrial environment, etc., each environment interacts and affects the internal ecological niche. Internal niche is the various dimensions of brand niche, including value niche [18], capital niche, human resources niche, technology niche, etc. Among them, the human resource niche is the core content of the internal niche, which reflects the learning and innovation ability of the enterprise. Similarly, the internal niche also interacts and affects the external environment. The external environment and the internal ecological niche form an ecosystem. The enterprise strengthens internal innovation by learning new knowledge and adapting to the new environment and finally acts on the external environment, which is the logic of outside-inside-outside.

(5) The motivation of the transition: external and internal. External factors are changing consumer perceptions, and internal factors are the leading role of heterogeneous human capital. From a philosophical point of view, the driving mechanism of the transition is heterogeneous human capital, and external factors further strengthen the speed and quality of the transition through internal factors. Therefore, it is necessary to emphasize heterogeneous human capital, that is, the main role of brand entrepreneurs. Brand entrepreneurs are composed of different subjects, and their knowledge, skills, and ability to learn knowledge and skills are affected by different levels of culture. The comprehensive role of property rights, systems, and culture forms the brand entrepreneur's pioneering and innovative spirit and the character of hard work and constant learning of new knowledge. Brand entrepreneurs continue to learn and innovate consumers' dynamic perceptions of brands. Driven by heterogeneous human capital, they generate incremental incentives to achieve brand ecology through the integration of external environment and internal niche, positive reinforcement mechanism, and self-organization mechanism. Bit transition.

(6) Constructivism of institutional change: North affirmed the importance of institutional change and believes that the system is of great significance to innovation. From the connotation point of view, system changes are divided into mandatory system changes and induced system changes. Inductive institutional changes emphasize the guiding role of institutional design entities (enterprises, industry associations, administrative agencies, etc.) in the transition of brand niche. At the same time, the transition of brand niche is the result of market allocation resources selected by different environments. The integration of the two is human the interactive integration of subjective initiative and environmental choices is a combination of brand planned economy and brand market economy. The mandatory system change reduces the efficiency of the transition and has a negative effect on the brand niche. The way of construction is the conscious and purposeful activity of the system design subject.

(7) Energy layer: interference factors and their characteristics. The state of transition is the process of absorbing energy, and the energy layer is the final hurdle that hinders the brand niche to complete the jump. The interference factors that constitute the energy layer are external factors and internal factors. The external factors are main competitors and the market. According to Porter's Five Forces Model, competitors are divided into alternative brands, existing competitors, and potential competitors. The characteristics are substitution, competitiveness, and latency. Substitution and competitiveness are the direct threats of alternative brands to the transition of brand niche, and latency is the indirect threat caused by potential competitors. The market element is the bargaining power of suppliers and customers, 
both of which have long-term characteristics for maximizing personal benefits. Internal factors are mainly chaotic management mechanisms and a lack of short-term tactics. The chaos of the management mechanism is the offside and absence of the organization, leadership, decisionmaking, planning, and control formed internally by external interference factors. The lack of short-term tactics is that the next step for the company is still unclear, and it only pursues quick success. The two factors are typical comprehensive complications in the process of brand niche transition, and both are harmful.

The transition model is divided into two parts: the first part is the evolution process of the transition, and the second part is the transition model. [Figure 1] is the first part, reflecting the evolutionary process of the transition. It is composed of the original brand niche-consumer perception-heterogeneous human capital-brand ecosystem-internal mechanism-path selectionnew brand niche. From the perspective of the model as a whole, the types of transition and evolution process can be divided: natural evolution and artificial catabolism. From the perspective of historical evolution and self-organization mechanism, it is the first type-natural evolution. This path assumes that competition is neutral. The brand niche is a natural evolution process from birth, growth, maturity, and decline with the environment. The second type is the implementation of an active construction plan under the conditions of natural evolution. It is the brand planned economy built on the brand market economy, and it is the interactive integration of plan-oriented and market-oriented. At the same time, through the interaction and integration of the external environment and the internal niche, the various dimensions of the internal niche are further strengthened, so that the brand niche is evolved. The mechanism is a joint force mechanism of the positive reinforcement mechanism and the self-organization mechanism.

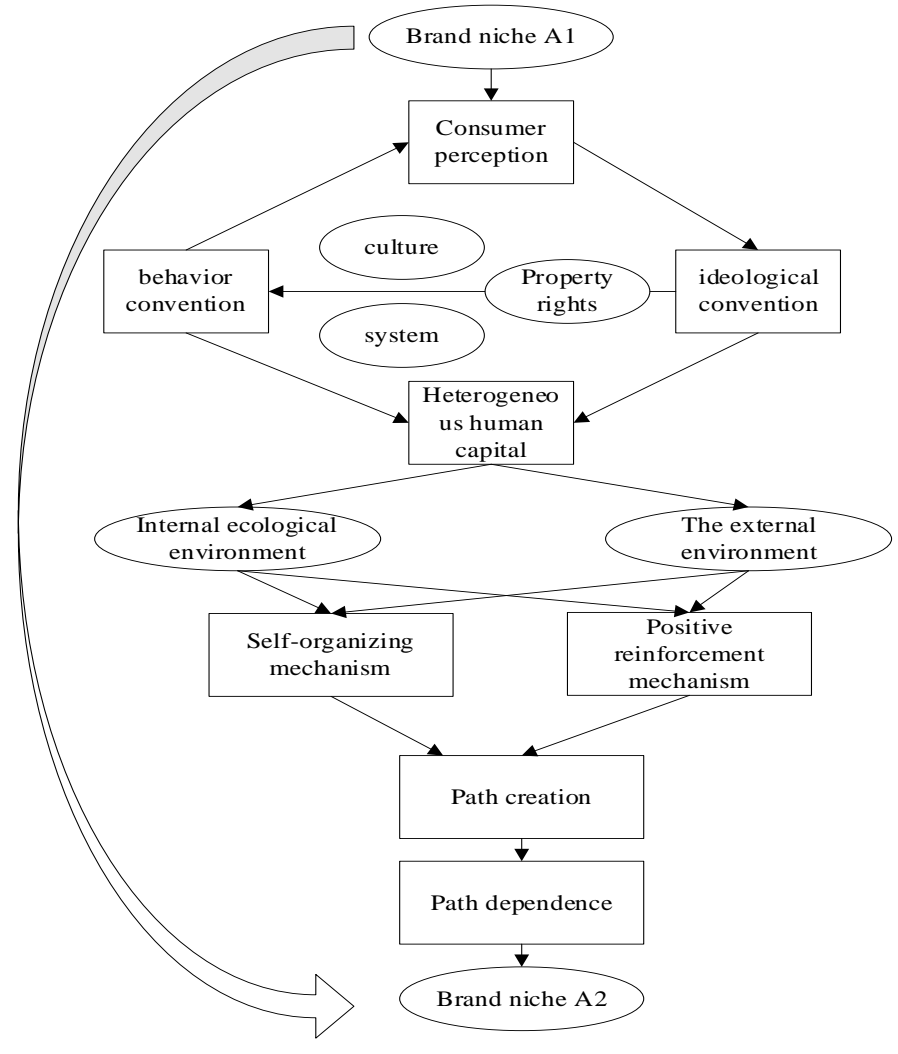

Figure 1. The evolution of transitions 
The second part is the transition model [Figure 2]. It is composed of the evolution form of brand niche A1 to An, the transition mode from An to B1, and the transition mode from B1 to $\mathrm{C} 1$. The first is the A1 to An stage, which is the continuous evolution process of the brand niche before the first transition. This process is relatively healthy and is the result of the positive interaction between the brand entrepreneur and the internal and external environment. The second is the stage from An to B1. This stage has to go through the energy layer, which is manifested as an unstable state when An transitions to B1. The dilemma of the transition is the result of internal chaos and incoordination between internal and external. The third is the stage from $\mathrm{B} 1$ to $\mathrm{C} 1$. From the perspective of the niche form, it is a continuous transition, that is, the brand niche transitions from the primary to the advanced. After natural evolution and artificial catalysis and continuous circulation, the brand niche is advanced.

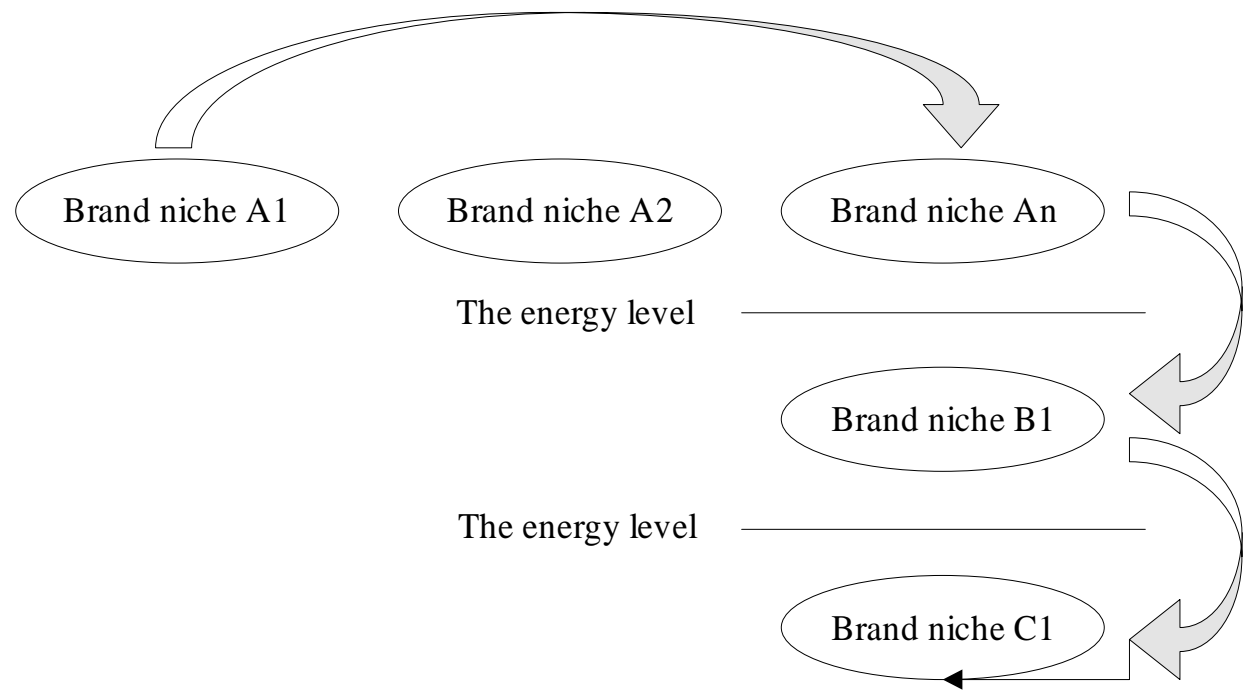

Figure 2. The transition models

\section{Case analysis}

Apple is a high-tech company founded by the Jobs team in 1976. As of 2018, the company's business involves personal computers, media players, smartphones, and tablets. In 2007, Apple gave birth to its first smartphone, iPhone, followed by a series of iPhone products. From the data point of view, the global market share of the iPhone was $11.9 \%, 13.1 \%, 14.2 \%$, and $17.2 \%$ in 2009, 2010, 2011, and 2012, respectively. It dropped in 2013 and 2014 and continued to grow after 2015. Regarding how the iPhone achieves the niche transition, this article conducts an empirical investigation based on the dual perspectives of consumer cognition and evolution (due to the availability of data, the empirical process can only partially explain the reasons for the transition, and the other part is explained by text).

\subsection{Construction of indicator system}

Relying on the mobile phone industry to build a brand niche indicator system, and selecting the leader of the mobile phone industry, Apple's mobile phone as the research object, it hopes to discover the key driving factors for Apple's niche transition in the past 10 years and provide guidance for the construction of other mobile phone brands. This study is mainly divided into two steps to construct the index system: First, the existing literature is screened according to 
the three levels of product elements, brand elements, and industrial elements, and a preliminary index system is obtained. Secondly, the selected index system is provided to the expert group for secondary screening and the index factors are appropriately increased. The research group and the expert group explain the meaning of each factor to form the final index system. Because the research object of this research is consumers, we select the sellers who know the consumer's perception best to form a focus group, including 4 "experts" from Apple retail stores, 3 salespeople from Samsung stores, and 3 Huawei dealer sales clerks in the shop. Finally, submit the determined indicator system to the expert group again, and let the experts score each indicator to determine the weight of each factor.

The product level includes five factors: "quality perception, performance perception, appearance evaluation, functional innovation, and symbolic attributes". Quality perception is a consumer's subjective perception of brand product quality. It has a certain influence on consumers' brand satisfaction, word-of-mouth communication, purchase intention, etc. It is of great significance to managers' brand building, so this article will It is incorporated into the product-level indicators of the mobile phone brand niche. Performance perception is a factor that describes consumers' subjective judgments and feelings about the performance of mobile phones. Studies have confirmed that the standby performance of mobile phones affects consumers' purchasing needs. The appearance evaluation factor reflects the fashion level of the mobile phone. This study believes that appearance is an important factor that affects consumers' perceptual needs and is one of the niche factors of mobile phones at the product level. Functional attributes and symbolic attributes are two important dimensions of product attributes [19]. Drawing on the research of existing scholars, this article believes that the two-dimensional attributes of the mobile phone industry should be functional innovation attributes and symbolic attributes. As a technological product, mobile phones generally have insufficient functions to attract consumers. Only when innovative elements are added can the functional attributes play a role. Therefore, innovation is added to the functional attributes to form a functional innovation factor. Also, symbolic attributes are becoming more and more important in the mobile phone industry. This can be reflected in the strategies of major brands to build expensive flagship phones. Therefore, this article believes that symbolic attributes are also important factors influencing the niche of mobile phones.

The brand level includes four factors: "brand attitude, logo recognition, brand reputation, and brand experience". Brand attitude is the comprehensive evaluation of consumers on the brand [20], and it is an important element of brand loyalty. Existing studies have shown that brand attitude has an important effect on word-of-mouth communication, purchase intention, etc., and is an important indicator for corporate managers to consider brand management. Logo recognition is the consumer's recognition of brand logos, the carrier of brand culture, spirit, and values, and one of the important indicators of consumer brand value recognition. Brand reputation is the ability of consumers to recognize a brand, and Aaker regards it as one of the important dimensions in his five-dimensional model of brand equity and ten-factor model [21]. Brand experience explains the influence of brand-related stimulus factors on consumer perception. As a currently popular marketing paradigm, brand experience plays an important role in strengthening consumer loyalty [22], enhancing brand equity, and building a strong brand. Based on the above analysis, this article believes that the four factors of "brand attitude, logo recognition, brand reputation, and brand experience" are important factors that affect consumers' brand awareness.

The industry level covers the three factors of "market position, technological change, and industry competition". The market position factor reflects the brand's leading position in a specific product category, and the driving factor behind it is the recognition of many customers. 
Technological change is another important factor affecting brand niche. The expert group believes that every technological change in the mobile phone industry is a "big wave", and whether an enterprise can catch the wave of technological change determines its state of existence. Industry competition mainly reflects the intensity of brand competition in the "community" of mobile phones. The fiercer the competition, the lower the degree of brand specialization, the wider the niche breadth, and the higher the degree of overlap, which directly affects the migration of the brand niche [10]. Based on the above explanations, this study believes that the three factors of "market position, change, and industry competition" are important factors that affect the brand niche.

The second opinion gathering is to determine the factor weight. In this study, a 5-level Likert scale questionnaire was designed, and 17 experts were asked to score 12 factors. The scores were 9 points, 7 points, 5 points, 3 points, and 1 point, corresponding to the very important, important, and important points in the questionnaire in turn. Five options are general, unimportant, and very unimportant. Bring the collected data into the following formula to calculate the preliminary factor weights. After normalization, the final 12 evaluation factor weights are $0.0962,0.1077,0.0814,0.0995,0.0551,0.0600,0.0929,0.0781,0.0798,0.0715$, $0.0962,0.0814$.

$$
\begin{gathered}
W=\left(\frac{v_{1}}{\sum_{i=1}^{n} V i \sum_{i=1}^{n} v_{i}}, \ldots, \frac{v_{n}}{\sum_{i=1}^{n} v_{i}}\right) \\
V_{i}=\frac{1}{k} \sum_{j=1}^{k} v_{i j}
\end{gathered}
$$

Among them, $\mathrm{W}$ is the factor weight, $\mathrm{n}$ is the number of evaluation indicators, $\mathrm{v}_{\mathrm{ij}}$ represents the score of the $\mathrm{j}$-th expert on the $\mathrm{i}$-th factor, and $\mathrm{k}$ is the number of experts.

\subsection{Data collection, processing, and analysis}

To reflect the evolution of the transition history, this article uses Apple's products over the years as a carrier to investigate consumers' evaluations of products in various years, to reflect the changes in the cognitive niche of Apple's mobile phone consumers from the side, thus explaining the evolution of the Apple brand. The process and the key elements that drive its transition provide a new perspective and theoretical basis for the mobile phone brand's strategic brand management. The research adopted a questionnaire survey to provide the survey participants with the photos and parameters of the six products of iPhone3 (2008), iPhone4 (2010), iPhone5 (2011), iPhone6 (2012), iPhone7 (2014), and iPhone XR (2018). At the same time, the interviewees are required to fill in the questionnaire after understanding the above material information.

Based on the 12 niche factors in [Table 1], the consumer questionnaire is designed: The first part is the basic information of the interviewee, mainly including the interviewee's gender, educational background, age, and other basic information. The second part is about consumers' evaluations of iPhone3 (2008), Phone4 (2010), iPhone5 (2011), iPhone6 (2012), iPhone7 (2014) and iPhone XR (2018). The questionnaire adopts a 5-level component system with scores of "5, 4, 3, 2, 1". A total of 279 questionnaires were distributed and 262 valid questionnaires were returned. Among them, 143 were male respondents, accounting for $54.58 \%$, and 119 were female respondents, accounting for $45.42 \%$. In terms of age, 59 people are 20 years old and below, accounting for $22.52 \%$; 61 people are $21-25$ years old, accounting for $23.28 \%$; 48 people are 26-30 years old, accounting for $18.32 \%$; 55 people are $31-35$ years 
old, accounting for $20.99 \%$; 36 years old and above 39 people, accounting for $14.89 \%$. In terms of academic qualifications, 67 people have a high school, technical secondary school or below, accounting for $25.57 \%$; 101 have a junior college, accounting for $38.55 \%$; 75 have a bachelor's degree, accounting for $28.63 \%$; 19 have a graduate degree or above, accounting for $7.25 \%$. In terms of personal annual income, 122 people with less than 40,000-yuan, accounting for $46.56 \%$; 35 people with $40,000-60,000$-yuan, accounting for $13.36 \%$; 48 people with 60,000 80,000 -yuan, accounting for $18.32 \%$; 35 people with 80,000-100,000-yuan, Accounting for $13.36 \%$; 22 people with more than 100,000-yuan, accounting for $8.4 \%$. This article sums up the scores of a certain product on a certain indicator and compares the scores on the symbolic attributes (including the two test items of prestige and self-expression) and the brand attitude (including the two test items of trust and satisfaction). Add the average value to get the comprehensive score of the 6 major products on the 11 major factors. The cognitive niche evaluation and comprehensive weights of the six major products are shown in [Table 1].

Table 1. The ecological niche system of mobile phone consumers' brand recognition

\begin{tabular}{|c|c|c|}
\hline First-level index & Secondary indicators & Word meaning interpretation \\
\hline \multirow{4}{*}{ Product level } & Quality perception & Durability \\
\cline { 2 - 3 } & Performance perception & $\begin{array}{c}\text { Running smoothness, heat dissipation, standby } \\
\text { time }\end{array}$ \\
\cline { 2 - 3 } & Appearance evaluation & fashion design \\
\cline { 2 - 3 } & Functional innovation & $\begin{array}{c}\text { Voice function, fingerprint recognition, } \\
\text { operating system }\end{array}$ \\
\cline { 2 - 3 } & Symbolic attributes & Identity, status, personality, image, etc. \\
\hline \multirow{3}{*}{ Brand level } & Logo identity & Signs, marks, symbols, etc. \\
\cline { 2 - 3 } & Brand reputation & Reputation \\
\cline { 2 - 3 } & Brand attitude & Cognition, emotion \\
\cline { 2 - 3 } & Brand experience & Consumer experience, customer experience \\
\hline Industry-level & Brand status & leadership \\
\hline
\end{tabular}

Using SPSS22.0 to process the validity and reliability of the questionnaire, the Cronbach's $\alpha$ values of the six products are $0.944,0.898,0.720,0.887,0.912,0.857$, which are all greater than the prevailing standard 0.7 , indicating that the internal consistency of the measurement items is good, the reliability is high. The KMO values were $0.974,0.949,0.801,0.935,0.957$, and 0.924 , which were all greater than 0.8 , indicating that the questionnaire data was highly valid.

This article divides the niche into 4 levels, namely: A (86-100), B (71-85), C (56-70), and D (under 55). From the perspective of factor weights, the five factors of "quality perception, performance perception, functional innovation, brand reputation, and technological change" are important indicators that affect the mobile phone niche. The five factors of "appearance evaluation, brand attitude, brand experience, position perception, and market competition" are in the second echelon of the element system. The influence of the two factors of "symbol attribute and logo identity" is relatively small.

As shown in [Table 2], Apple's mobile phones have gone through three main stages: the first stage is iPhone3 (2008), Phone4 (2010) to iPhone5 (2011), this stage reflects the vertical transition process (C-BA) as a whole. The second stage is from iPhone5 (2011) to iPhone6 (2012) (A-B). This stage is the niche fluctuation stage of Apple's mobile phone. The third stage 
is iPhone6 (2014), iPhone7 (2014), and iPhone XR (2018). This stage reflects the process of Apple's mobile phone niche consolidation (B-B-A). From the perspective of the motivation of the transition, quality perception, performance perception, functional innovation, and brand reputation are the main factors. One is that quality perception, performance perception, and brand reputation belong to the category of consumer cognition and change over time. The change process jumps greatly in the vertical transition segment. For example, the quality perception increases from 65.235 (iPhone3) to 91.887 (iPhone5). The horizontal transition segment is relatively smooth, for example, the performance perception increases from 88.339 (iPhone6) to 91.335 (iPhone XR). The second is that functional innovation is a manifestation of the dynamic innovation of brand entrepreneurs. As we all know, Apple's R\&D strategy is the core strategy. The third is the cross-relationship between quality perception, performance perception, brand reputation, and functional innovation. Apple focuses on brand communication with consumers to form value co-creation. If users have purchased their products, Apple will regularly send them formal and respectful e-mails with questionnaires, or users or investors can log on to the homepage to ask questions or write to you, such as your recent purchase of Apple Any comments and suggestions on the product? Is your online i-tunes smooth? Is there a usage problem? Has it been resolved? Have you participated in i-genius classroom activities? Any suggestions for this? From the perspective of constructivism, the US government introduced policies in 2012 to support the chip manufacturing carried by Apple, in 2013 it introduced policies to restrict Samsung's sales in the United States, and in 2017 it implemented a tax reduction and exemption of US\$207.8 million for Apple. These policies are all within a certain range. To a certain extent, promote the leap of the iPhone niche. From the perspective of heterogeneous human capital, Apple is a multinational company with technology and marketing talents from the United States, Japan, Germany, France, and other countries to build its brand. From the perspective of the development of brand entrepreneurs, the cultural aspect is the embodiment of symbolic attributes and Peugeot identity. In terms of property rights, Apple encourages employees to hold stocks and grants more employees stock options through a new equity incentive plan after 2015. The institutional aspect is a good institutional environment in the United States. From the perspective of the brand ecosystem, due to limited data, the external environment only lists the competitive environment and technological changes. From the perspective of the energy layer, Apple faces strong competitors such as Huawei, Xiaomi, Samsung, etc., and internal decision-making errors, such as the "speed-down gate" incident that occurred at the end of 2017, will hinder the niche transition. The second stage is the fluctuation stage of Apple's mobile phone niche. The iPhone has been gradually overtaken by competitors such as Huawei and Samsung in terms of the four major driving factors of quality perception, performance perception, functional innovation, and brand reputation. Apple has gradually shifted its focus to "symbol value, logo recognition, brand status" and other driving factors. Leading to it gradually becoming a luxury, a status symbol rather than the "leader" of technological innovation in the mobile phone industry. The third stage is the stabilization and improvement of the niche of Apple's mobile phones. Although the niche level of iPhone XR is A, iPhone6 to iPhone XR are generally stable. The iPhone XR released in 2018 has made breakthroughs in multiple functions, re-stabilizing the brand's niche. 
Table 2. Consumer cognitive niche evaluation of Apple products

\begin{tabular}{|c|c|c|c|c|c|c|c|}
\hline & Weight & iPhone3 & iPhone4 & iPhone5 & iPhone6 & iPhone7 & iPhone XR \\
\hline Quality awareness & 0.096 & 65.235 & 80.822 & 91.887 & 81.496 & 68.41 & 83.132 \\
\hline Performance perception & 0.108 & 84.03 & 89.524 & 105.037 & 88.339 & 76.381 & 91.355 \\
\hline Appearance evaluation & 0.081 & 56.013 & 69.121 & 80.519 & 67.818 & 54.629 & 69.854 \\
\hline Function innovation & 0.1 & 66.271 & 81.297 & 94.332 & 83.586 & 70.351 & 84.481 \\
\hline Symbol attribute & 0.055 & 37.467 & 46.007 & 52.895 & 45.759 & 38.817 & 45.622 \\
\hline Sign recognition & 0.06 & 40.522 & 52.469 & 56.971 & 49.887 & 42.863 & 50.788 \\
\hline The brand name & 0.093 & 60.961 & 79.546 & 87.259 & 79.36 & 65.235 & 80.289 \\
\hline Brand attitude & 0.078 & 53.867 & 64.727 & 76.406 & 64.688 & 54.18 & 66.68 \\
\hline Brand experience & 0.08 & 54.722 & 66.448 & 80.727 & 67.884 & 56.317 & 68.283 \\
\hline Brand position & 0.072 & 49.796 & 61.387 & 66.752 & 58.453 & 51.227 & 60.027 \\
\hline Technological change & 0.096 & 65.813 & 81.688 & 92.657 & 78.609 & 67.833 & 83.613 \\
\hline Industry competition & 0.081 & 54.385 & 55.85 & 68.225 & 67.167 & 80.275 & 75.308 \\
\hline Total value & 1 & 689.082 & 828.886 & 953.669 & 833.046 & 726.519 & 859.431 \\
\hline Niche level & & C & B & A & B & B & A \\
\hline
\end{tabular}

\section{Conclusion}

Based on the theory of brand ecology and evolutionary economics, this paper constructs a model of the transition path of brand niche and clarifies the driving mechanism of the transition of brand niche from the perspective of consumer cognition, that is, brand entrepreneurs under the leading role of heterogeneous human capital Bring out the innovative spirit of brand entrepreneurs through the three elements of property rights, system, and culture, and follow satisfactory decisions to search for consumer cognition to form value co-creation (value niche). This process is accompanied by historical evolution and constructivism. It strengthens the interaction and integration of the internal niche (technical niche, resource niche, etc.) with the external environment through the value niche, and promotes the enhancement of various dimensions of the brand niche, thereby enabling the brand niche to leap. Relying on the mobile phone industry, a consumer cognitive niche system has been constructed and the importance of various factors affecting the industry niche has been identified. Specifically, the mobile phone brand niche system is composed of 12 factors at the product level, brand level, and industry level. Among them, the key factors mainly include "quality perception, performance perception, functional innovation, brand reputation, and technological change." The high factor weight is a key factor that affects consumer brand perception and a key indicator that determines the transition of the ecological niche. Taking the Apple mobile phone as a specific case, the niche at each time point was measured and the niche transition was proved to be a historical process, and the comprehensive evaluation scores of the niche of 6 mobile phones were calculated and ranked. On the whole, it showed a trend of rising first and then stabilizing. Specifically, the first stage is iPhone3 (2008), Phone4 (2010) to iPhone5 (2011), reflecting the vertical transition process of Apple's mobile phone niche (C-B-A); the second stage is iPhone5 (2011) to iphone6 (2012) (A-B) is the stage of fluctuations in the niche of Apple's mobile 
phone; the third stage is from iPhone6 (2014), iPhone7 (2014) to iPhone XR (2018), reflecting the steady recovery of the niche of Apple's mobile phone (B-B) -A).

\section{References}

[1] N. Maksymenko, "The psychological predictors of activity of IT professional sin conditions of organizational transition to remote work," Bulletin of Taras Shevchenko National University of Kyiv Series "Psychology", vol.2, no.9, pp.38-42, (2018)

[2] M. Jovanović, A. Mas, A. L. Mesquida, and B. Lalic "Transition of organizational roles in agile transformation process: A grounded theory approach,” Journal of Systems and Software, Nov., vol.133, pp.174-194, (2017)

[3] P. O. Gartland and B. J. Slagsvold, "Transitions conserving parallel momentum in photoemission from the (111) face of copper," Physical Review B, vol.12, no.10, pp.4047-4058

[4] O. Mtapuri, A. Giampiccoli, and S. Jugmohan, "Marketing community-based tourism ventures: Pathways in a marketing route model," Journal for New Generation Sciences, vol.15, no.1, pp.385-401, (2017)

[5] C. Jessica, C. Suhana, and K. Prachi, "Temporal variations in cigarette tobacco bacterial community composition and tobacco-specific nitrosamine content are influenced by brand and storage conditions," Frontiers in Microbiology, vol.8, no.2757, pp.358, (2017)

[6] C. Gebhard, "EU crisis management coherence from an institutional perspective," Management Learning, vol.26, no.1, pp.73-90, (2018)

[7] H. Watson, R. L. Finn, and K. Wadhwa, "Organizational and societal impacts of big data in crisis management," Journal of Contingencies and Crisis Management, vol.25, no.1, pp.15-22, (2017)

[8] A. Winkler, "Wrap -speed branding: The impact of technology on marketing," Hoboken: Wiley

[9] M. T. Hannan, R. C. Glenn, and P. Laslo, "The organizational niche," Sociological Theory, vol.21, no.4, pp.309-341, (2003)

[10] W. Dong, X. Zhang, and X. Liu, "Evaluating the consistency of current mainstream wearable devices in health monitoring: A comparison under free-living conditions,” Journal of Medical Internet Research, vol.19, no.3, pp.68, (2017)

[11] W. Jarvis and S. Goodman, "Effective marketing of small brands: Niche positions, attribute loyalty, and direct marketing," Journal of Product and Brand Management, vol.14, no.5, pp.292-299, (2005)

[12] T. Foydel, "Brand ecosystem management," Contract Manufacturing and Packaging, vol.22, no.1, pp.40-41, (2008)

[13] A. Holmquist, M. Magnusson, and M. Livholts, "Reinventing tradition: Exploring the creation of new meaning through innovations involving craft-based design," Creativity and Innovation Management, vol.28, no.1, (2019)

[14] T. Beuzen and J. Simmons, “A variable selection package driving Netica with python," Environmental Modelling and Software, no.115, pp.1-5, (2019)

[15] J. Aaker, "Dimensions of brand personality," Journal of Marketing Research, vol.34, no.3, pp.347-356

[16] F. Aydın, "Willingness to communicate (WTC) among intermediate-level adult Turkish EFL learners: Underlying factors," Journal of Qualitative Research in Education, vol.5, no.3, pp.1-29, (2017)

[17] R. Cao, "An analysis on the brand ecosystem of Nomads chain of restaurants in Mongolia," Journal of Inner Mongolia University of Finance and Economics, (2019)

[18] V. A. Zeithaml, "Consumer perceptions of price, quality, and value: A means-end model and synthesis of evidence," Journal of Marketing, vol.52, no.3, pp.2-22

[19] K. E. Voss, E. R. Spangenberg, and B. Grohmann, "Measuring the hedonic and utilitarian dimensions of consumer attitude, Journal of Marketing Research, vol.40, no.3, pp.310-320, (2003)

[20] K. L. Keller, “Conceptualizing, measuring, and managing customer-based brand equity,” Journal of Marketing, vol.57, no.1, pp.1-22

[21] D. A. Aaker, "Managing brand equity: Capitalizing on the value of a brand name," New York: Google Scholar 
Leap Forward Path Model of Niche based on Brand Ecological Theory

[22] Y. Deliana, and I. A. Rum, "Understanding consumer loyalty using neural network," Polish Journal of Management Studies, vol.16, no.2, pp.51-61, (2017) 